\title{
土壤氮磷添加下豆科草本植物生物固氮与磷获取 策略的权衡机制
}

\author{
李 强 ${ }^{*}$ 黄迎新 周道玮丛山 \\ 中国科学院东北地理与农业生态研究所, 吉林省草地畜牧重点实验室, 长春 130102
}

摘 要 豆科草本植物的固氮是陆地生态系统重要的自然氮源输入方式, 影响着草地生产的经济性和可持续性。为探讨氮磷 交互作用影响豆科草本植物生物固氮率的潜在生理生态机制, 该研究选取 8 种豆科草本植物分别种植在对照、氮肥添加、磷 肥添加和氮磷耦合添加处理的土壤中, 进行野外盆栽实验。测定了初花期植物生物量和营养含量、根部碳水化合物含量、根 际 $\mathrm{pH}$ 、根际柠檬酸含量、根际有效磷含量、植物根瘤生物量、磷含量及其生物固氮率。主要结果: 依赖于豆科物种, 氮添加 显著促进了豆科草本植物根际磷的活化, 降低了根生物量分配以及根系非结构性碳水化合物含量。在两种磷添加处理下, 氮 添加导致 8 种豆科草本植物根瘤生物量平均下降 $27 \%-36 \%$, 生物固氮率平均下降 $20 \%-33 \%$ 。磷添加降低了根际的磷活化, 但 促进了豆科草本植物根系发育和非结构性碳水化合物的积累。在施氮和不施氮条件下, 磷添加分别使 8 种豆科草本植物的生 物固氮率提高了 $45 \%-69 \%$ 和 $0-47 \%$ 。氮添加降低豆科草本植物生物固氮率, 其原因是氮添加提高了植物磷需求, 为活化更多 磷, 豆科草本植物降低根系生物量和根系非结构性碳水化合物的含量, 导致根瘤发育受到限制。在氮添加的同时进行磷添加, 能够改善土壤氮磷平衡, 促进根系生长和非结构性碳水化合物积累, 缓解了增氮对生物固氮的抑制作用。

关键词 氮固定; 磷活化; 豆科饲草; 根际; 根瘤; 共生

李强, 黄迎新, 周道玮, 从山 (2021). 土壤氮磷添加下豆科草本植物生物固氮与磷获取策略的权衡机制研究. 植物生态学报, 45, 00-00. DOI: $10.17521 /$ cjpe.2020.0241

\section{Mechanism of the trade-off between biological nitrogen fixation and phosphorus acquisition strategies of herbaceous legumes under nitrogen and phosphorus addition}

LI Qiang ${ }^{*}$, HUANG Ying-Xin, ZHOU Dao-Wei, and CONG Shan

Northeast Institute of Geography and Agroecology, Chinese Academy of Sciences, Jilin Provincial Key Laboratory of Grassland Farming, Changchun 130102, China

\section{Abstract}

Aims Nitrogen fixation of herbaceous legumes is not only an important natural nitrogen input to terrestrial ecosystems, but also determines the economy and sustainability of grassland production. This study aimed to determine the underlying physiological and ecological mechanisms of the interaction between nitrogen $(\mathrm{N})$ and phosphorus $(\mathrm{P})$ on biological $\mathrm{N}$ fixation rate of legumes.

Methods In a pot experiment, eight species of herbaceous legumes were separately grown in soils with four treatments including no fertiliser, $\mathrm{N}$ addition, $\mathrm{P}$ addition, and both $\mathrm{N}$ and $\mathrm{P}$ (NP) addition. Plant biomass and nutrients concentrations, root carbohydrate concentration, $\mathrm{pH}$ in rhizosphere, citric concentration in in rhizosphere, avaiable $\mathrm{P}$ concentration in rhizosphere, root nodule biomass, $\mathrm{P}$ concentration in root nodule, and $\mathrm{N}$ fixation rate of these legume plants were examined.

Important findings Depending on legume species, $\mathrm{N}$ addition significantly increased relative rhizosphere $\mathrm{P}$ mobilization, but reduced investment in root biomass and the concentration of non-structural carbohydrate (NSC) in roots. Averaged results of $\mathrm{N}$ addition and NP addition treatments indicated that $\mathrm{N}$ addition caused $27 \%-36 \%$ decline in nodule biomass and $20 \%-33 \%$ decline in biological $\mathrm{N}$ fixation rate for the studied eight legume species. By contrast, $\mathrm{P}$ addition significantly promoted root development and NSC accumulation associated with decreasing relative rhizosphere $\mathrm{P}$ mobilization. Consequently, $\mathrm{P}$ addition increased the biological $\mathrm{N}$ fixation rate of the

收稿日期Received: 2020-07-17 接受日期Accepted: 2020-10-22

基金项目: 中国科学院战略先导专项(XDA23060403)和国家自然科学基金(31600318)。Supported by the Strategic Science and Technology Guide Project of CAS (XDA23060403), and the National Natural Science Foundation of China (31600318).

* E-mail: liqiang@iga.ac.cn 
eight legume species by $45 \%-69 \%$ and $0-47 \%$ with and without $\mathrm{N}$ fertilization, respectively. We concluded that $\mathrm{N}$ addition reduced biological $\mathrm{N}$ fixation rate via reducing root biomass and root NSC concentration and increasing rhizosphere P mobilization; P addition helped to improve soil N-P balance and promote root growth and NSC accumulation, which can alleviate the inhibition of biological $\mathrm{N}$ fixation by $\mathrm{N}$ fertilization.

Key words nitrogen fixation; phosphorus mobilization; legume forage; rhizosphere; root nodule; symbiotic

Li Q, Huang YX, Zhou DW, Cong S (2021). Mechanism of the trade-off between biological nitrogen fixation and phosphorus acquisition strategies of herbaceous legumes under nitrogen and phosphorus addition. Chinese Journal of Plant Ecology, 45, 00-00. DOI: $10.17521 /$ cjpe. 2020.0241

生物固氮是豆科草本植物的重要氮吸收途径, 豆科草本植物的生物固氮率不仅决定其同化大气氮 的总量(Augusto et al., 2013), 还通过改变土壤氮的 相对消耗影响豆科草本植物生长的氮经济( Li et al., 2016a, 2020)。外源氮输入和生物固氮的积累增加了 土壤中氮的有效性(Galloway, 1998; Vitousek et al., 2013)。然而, 增加的土壤氮有效性会潜在地降低豆 科草本植物的生物固氮率(Salvagiotti et al., 2008; Nyfeler et al., 2011), 进而阻碍豆科草本植物对生态 系统的进一步固氮输入。以往的研究表明, 豆科植 物能够根据土壤氮肥力状况调整在固定大气氮和获 取土壤氮方面的投资策略(Vitousek et al., 2002; West et al., 2005)。这些研究认为生物固氮的代谢成 本一般高于土壤氮吸收, 因此高土壤氮有效性下豆 科植物倾向于增加根系对土壤氮的吸收, 但抑制代 谢上高成本的根瘤发育和生物固氮(West et al., 2005)。这一观点暗示当氮有效性增加, 豆科植物生 物固氮被抑制将成为普遍现象。然而, 其他研究发 现, 外源氮的输入并不总是下调豆科植物生物固氮 率, 这意味着一些其他因素可能会改变氮富集对生 物固氮率的影响(Reed et al., 2007; Zheng et al., 2019)。

磷对植物生长和代谢具有关键调控作用(White \& Hammond, 2008; Png et al., 2017)。因为根瘤发育和 生物固氮过程的高磷消耗, 豆科植物对磷的供应尤 为敏感 (Batterman et al., 2013; Divito \& Sadras, 2014)。研究表明磷添加能够提高豆科植物根瘤发育 和生物固氮率(Augusto et al., 2013; Maistry et al., 2013), 尤其在磷资源限制环境中(Betencourt et al., 2012)。尽管氮、磷在土壤中有不同的生物地球化学 循环过程, 增加的氮供应很可能改变土壤内在的氮 -磷平衡(Elser et al., 2010; Peñuelas et al., 2012), 提 高植物磷需求, 驱动土壤磷的消耗(Güsewell, 2004; Png et al., 2017), 并最终加剧植物磷溃乏。基于此,
氮增加下, 改变的土壤氮磷供给平衡很可能成为驱 动豆科植物生物固氮率下降的额外机制。在这种情 景下, 土壤中磷肥的状态能够潜在改变氮富集对豆 科植物生物固氮的抑制作用。先前的研究发现当土 壤磷有效性较低时, 氮增加抑制根系结瘤, 但当土 壤磷有效性较高时, 氮增加能够促进根系结瘤 (Gates \& Wilson, 1974), 相似的结果也存在于其他 研究中(Gentili \& Huss-Danell, 2003)。这些研究已经 表明氮、磷在调控豆科植物固氮行为中的交互作用。 然而, 对于氮-磷平衡的改变如何驱动植物生理变 化, 并最终改变豆科植物根系结瘤和生物固氮率, 我们仍旧缺乏深入理解。

磷限制影响豆科植物根瘤细胞的构建, 并影响 生物固氮过程中的能量供应(Sulieman et al., 2013; Divito \& Sadras, 2014)。然而, 并不清楚氮增加导致 的相对磷贵乏是否已经经由降低植物和根瘤磷吸收 来抑制根系结瘤和生物固氮率。此外, 在磷资源限 制下, 许多陆地植物, 尤其豆科植物已经发展了物 种特异性的适应机制, 诸如强化植物-菌根互作、刺 激根际磷活化等来满足植物磷需求(Nuruzzaman, 2006; Zhou et al., 2009)。进一步的实验证据表明上 述磷获取策略的实现极大依赖于根系形态和生理的 改变(Nuruzzaman, 2006; Betencourt et al., 2012; Png et al., 2017)。然而, 氮增加是否激发了这些磷获取 策略, 并借此改变豆科植物根系生理, 导致生物固 氮率的下降不得而知。

本研究利用 8 种豆科草本植物作为栽培实验材 料, 通过氮磷交互添加实验, 探究氮磷交互作用影 响豆科植物生物固氮率的生理和生态机制。本文主 要阐明 2 个科学问题: 1)氮添加是否通过限制豆科草 本植物及其根瘤磷吸收降低其生物固氮率? 2)如果 豆科草本草植物磷吸收未受影响, 氮增加怎样刺激 豆科草本植物的磷获取策略来满足磷吸收, 并导致豆 科草本生理如何变化? 结果对生物固氮率有何影响? 


\section{1 材料和方法}

\section{1 研究地点}

室外盆栽实验在中国科学院长岭草地农牧研究 站 $\left(123.52^{\circ} \mathrm{E}, 44.55^{\circ} \mathrm{N}\right.$; 海拔 $145 \mathrm{~m}$ ) 执行。该地点 2000-2016年间, 年平均气温 $5.5{ }^{\circ} \mathrm{C}$, 年降水量 $404 \mathrm{~mm}$, $70 \%$ 以上的降水发生在 5-9月。土壤类型为草甸盐碱 土, 羊草(Leymus chinensis)为该区域优势植物物种。

\section{2 盆栽实验设计}

利用在松嫩草甸种植的 8 种豆科草本植物为实 验材料, 包括 4 个本地物种 (花苜宿 (Medicago ruthenica)、兴安胡枝子(Lespedeza davurica)、草木 樨(Melilotus officinalis)、野大豆(Glycine soja)), 以及 4 个引种物种(紫花苜莑(Medicago sativa)、黄花苜宿 (Medicago falcata)、杂花苜宿(Medicago varia)和百 脉根(Lotus corniculatus))。本研究使用该研究区天然 植被的优势物种, 并且和本研究中多数豆科草本植 物有共存关系的羊草作为豆科草本植物固氮评估的 参照植物。我们的前期实验表明使用羊草做参照植 物获得的豆科草本植物固氮评估数据相近于使用多 种参照植物获得的平均测量数据(Li et al., 2015)。所 有植物种子于 2016 年收集自中国科学院长岭草地农 牧生态研究站内的天然草地及豆科草本植物种子 田。2017年6-9月间, 所有植物被分别种植生长在无 渗漏的塑料花盆中 (高 $30 \mathrm{~cm}$, 直径 $25 \mathrm{~cm}$ )。实验土壤 收集自天然草甸0-30 cm工层, 过 $2 \mathrm{~mm}$ 篎后混匀。 每盆装入等量的实验土壤, 实验土壤的初始理化性 质为 $\mathrm{pH} 8.74$ 、有机质含量 $17.3 \mathrm{~g} \cdot \mathrm{kg}^{-1}$ 、总氮含量 1.26 $\mathrm{g} \cdot \mathrm{kg}^{-1}$ 、总磷含量 $0.31 \mathrm{~g} \cdot \mathrm{kg}^{-1}$, 植物化学计量学的研 究证据表明该研究位点土壤处于氮限制状态(宋彦 涛等, 2012)。植物种植前, 所有种子进行表面消毒, 然后先置于浅沙盘中发芽。待种子发芽后, 同一物 种 4 个发芽的种子被转移种植入一个花盆。为保障每 种豆科草本植物生长和固氮不受土壤中相应根瘤菌 的数量限制, 田间收集每种植物的新鲜根瘤, 采用 破碎的根瘤菌液对每种植物进行根瘤接种。

实验采取完全随机区组设计, 所有实验花盆按 重复分 3 组摆放, 每组内设置 8 个物种、 2 个氮添加水 平 $\left(0 、 14 \mathrm{~g} \cdot \mathrm{m}^{-2}, \mathrm{NH}_{4} \mathrm{NO}_{3}\right)$ 和 2 个磷添加水平 $\left(0 、 3.5 \mathrm{~g} \cdot \mathrm{m}^{-2}\right.$, $\left.\mathrm{Ca}\left(\mathrm{H}_{2} \mathrm{PO}_{4}\right)_{2} \cdot \mathrm{H}_{2} \mathrm{O}\right)$ 交互处理, 组内各处理花盆位置随 机摆放。相似气候区的研究认为 $14 \mathrm{~g} \cdot \mathrm{m}^{-2}$ 的氮添加 量足够解除本区植物生长的氮限制(Bai et al., 2010;
Zhan et al., 2017), 磷的添加率基于氮添加率和实验 土壤 $\mathrm{N}: \mathrm{P}$ 比例设置。氮磷肥料分 3 次溶解于水中施入 花盆。整个实验期间, 花盆露天放置, 隔绝降水。基 于花盆面积和当地正常降水量考量, 间隔3-4天, 每 盆植物浇灌 $1000 \mathrm{~mL}$ 水(相当于每隔3-4天接受 $20 \mathrm{~mm}$ 降水), 保证植物生长不受水分限制。为保证 实验过程中各处理植物所接受的光等资源随机一致, 重复组内花盆位置每周随机调换一次。

\section{3 测量和取样}

参考邹长明等(2015)的研究, 当每种豆科草本 植物生长至初花期(近于豆科草本植物最大地上生 物量时期), 进行收获取样。取样时, 将长有植物的 完整土壤与花盆小心分离。然后, 将土壤轻柔敲碎 并分离携带根际土壤的植株。本研究中, 根际被定 义为距根表面 $2 \mathrm{~mm}$ 范围内的土壤, 这一范围被认为 是豆科植物主要的根系磷吸收区(Hinsinger，1998; Nuruzzaman et al., 2006)。使用一个柔软的小毛刷细 致收集粘附在根上的根际土壤。每盆中所有植物的 根际土壤组合为一个样品。然后, 将分离的植物个 体在水龙头下小心清洗根部。然后, 将植株分为地 上、根部和根瘤 3 部分, 查数根瘤数量。每盆随机选 取1株植物的新鲜根系材料进行菌根侵染和根系磷 酸酶活性检验。余下的植物材料在 $60{ }^{\circ} \mathrm{C}$ 下分别烘 干、称质量。每个花盆中, 一株植株的所有部分组 合并粉碎用于 ${ }^{15} \mathrm{~N}$ 同位素分析。每盆植物剩余地上、 根系和根瘤样品分类合并粉碎, 部分样品用于分析 氮磷含量, 根系样品同时用于分析非结构性碳水化 合物含量。植物和根际土壤取样后, 将非根际土壤 混合均匀后取样。

\section{4 植物土壤分析}

为测量菌根侵染率, 依据 $\mathrm{Wu}$ 等(2015)的方法, 部分根被切为 8 个 $1 \mathrm{~cm}$ 长根段, 经净化、软化、酸化、 染色和去色等步骤后压片镜检。菌根侵染率根据下 面公式计算:

菌根侵染率 $(\%)=($ 侵染根长度 $/$ 总镜检根长度 $) \times 100(1)$

羊草和豆科草本植物的 ${ }^{15} \mathrm{~N}$ 同位素丰度使用 ThermoFisher MAT253 同位素比质谱仪(ThermoFisher, Waltham, USA)分析。总氮含量采用凯氏定氮 法(Sparks et al., 1996)测定, 总磷含量通过过硫酸氧 化消解后采用分光光度计测定(Schade et al., 2003)。 非结构性碳水化合物 (NSC) 含量依据 Buysse 和 Merckx (1993)改进的比色法测定。部分根际土壤经 
由 $0.2 \mu \mathrm{mol} \cdot \mathrm{L}^{-1} \mathrm{CaCl}_{2}$ 溶液浸提, 取悬浮液冷冻于 $-20{ }^{\circ} \mathrm{C}$ 。悬浮液经 $100{ }^{\circ} \mathrm{C}$ 水浴 $1 \mathrm{~h}$, 然后在 $3500 \mathrm{r} \cdot \mathrm{min}^{-1}$ 下离心 $10 \mathrm{~min}$, 再经 $0.45 \mu \mathrm{m}$ 滤膜过滤, 然后, 使用 离子色谱测定柠檬酸含量(Baziramakenga et al., 1995)。剩余根际和非根际土壤样品于 $50{ }^{\circ} \mathrm{C}$ 下烘干, 然后研磨过 $0.2 \mathrm{~mm}$ 篮, 土壤有效磷含量经 $\mathrm{NaHCO}_{3}$ 溶液浸提后根据Olsen等(1954)的方法测定。根际土 壤经超纯水浸提后采用PHS-25 pH计测定。部分非 根际土壤样品冷冻于 $-20{ }^{\circ} \mathrm{C}$, 然后经 $2 \mathrm{~mol} \cdot \mathrm{mol}^{-1}$ $\mathrm{KCl}$ 溶液浸提后, $\mathrm{NH}_{4}{ }^{+}$和 $\mathrm{NO}_{3}{ }^{-}$含量使用Bran-Luebbe AA3自动分析仪(Bran-Luebbe, Hamburg, Germany) 测定(Miller \& Keeney, 1982)。所有养分含量特征基 于土壤干质量计量。

\section{5 数据计算和统计分析}

豆科草本植物组织中生物固氮率(BNF, \%)采用 如下公式计算(Unkovich et al., 2008):

$$
B N F=100 \times\left(\frac{\delta^{15} \mathrm{~N} \text { 羊草- } \delta^{15} \mathrm{~N} \text { 豆科草本植物 }}{\delta^{15} \mathrm{~N} \text { 羊草-B }}\right)(2)
$$

式中, $\delta{ }^{15} \mathrm{~N}$ 表示植物组织相对大气的 ${ }^{15} \mathrm{~N}$ 原子丰度 变化。 $B$ 代表豆科草本植物组织完全依赖大气氮生 长时的 $\delta{ }^{15} \mathrm{~N}$ 。本研究采用整株植物材料来评估生物 固氮, 这时整株植株的 ${ }^{15} \mathrm{~N}$ 丰度应与大气一致, 所以 B等于0 (Unkovich et al., 2008)。根际磷活化效应定 义为根际土壤有效磷含量与非根际土壤有效磷含量 的比值(Zhan et al., 2017)。

所有数据在统计前先进行正态分布和方差齐性 检验。采用混合线性模型分析氮磷添加及其交互作 用对土壤养分特征的影响, 其中, 氮、磷添加及其交 互作用作为固定效应, 豆科物种作为随机效应。采 用一般线性模型分析氮、磷添加、物种及其交互作 用对其他测量指标的影响。多重比较通过邓肯检验 (Duncan's test) 实现。每个处理下所有 8 个豆草物种 每个重复的平均值被用于进一步的回归分析, 检验 根生物量、根碳水化合物含量和根系菌根侵染率及 根际化学的相关性, 并检验根生物量、根碳水化合 物含量、根际磷活化和豆草生物固氮率的相关关系。 统计显著性水平设置为 $p<0.05$ 。所有数据分析通过 SPSS 17.0实现。

\section{2 结果}

\section{1 土壤氮磷有效性}

氮添加使土壤有效氮含量平均增加 $93 \%$, 并促
使土壤有效氮磷比显著提高, 而磷添加促使土壤有 效磷含量平均增加 $82 \%$, 并导致土壤有效氮磷比显 著下降(表1)。

\section{2 植物生物量和养分含量}

平均两个磷添加处理, 氮添加促使 8 种豆科草 本植物地上生物量增加 $25 \%-44 \%$ (图1A)。在无氮和 氮添加下, 磷添加分别促使不同豆科草本植物根生 物量增加 $11 \%-31 \%$ 和 $20 \%-36 \%$ (图1B)。综合两个磷 添加处理, 氮添加促使 8 种豆科草本植物氮含量提 高 $14 \%-26 \%$, 综合两个氮添加处理, 不同豆科草本植 物氮含量在磷添加后的变化范围为 $-7 \%-9 \%$ (图 $2 \mathrm{~A}$ )。 依赖于豆科物种，氮添加对豆科草本植物磷含量的 作用从降低6.7\% (紫花苜宿)到增加 5\% (兴安胡枝 子), 两种氮添加处理下, 磷添加促使 8 种豆科草本 植物磷含量提高 $10 \%-22 \%$ (图2B)。不同豆科草本植 物氮磷比的变化与土壤有效氮磷比保持一致(图2C)。

\section{3 根际磷活化、根生理和根际化学}

在无磷和磷添加下, 氮添加分别导致 8 种豆科 草本植物根际磷活化增加 $15 \%-33 \%$ 和 $7 \%-21 \%$, 综 合两个氮添加处理, 磷添加促使 8 种豆科草本植物 根际磷活化降低 $37 \%-41 \%$ (图3A、3B)。在无磷或磷 添加下, 氮添加使豆科草本植物根部非结构性碳水 化合物含量平均降低 $10 \%$ 或增加 $3 \%$, 不同豆科草本 植物根非结构性碳水化合物含量对氮添加的响应存 在差异。综合两个氮添加处理, 磷添加促使 8 种豆科 草本植物根部非结构性碳水化合物含量增加 $25 \%-42 \%$ (图4A)。氮添加对豆科草本植物根系菌根 侵染率无显著作用, 而磷添加降低豆科草本植物根 系的菌根侵染率(图4B)。无磷/磷添加下, 氮添加分 别促使 8 种豆科草本植物根际土壤 $\mathrm{pH}$ 下降 $0.52-0.80$ 或0.03-0.37 (图4C), 磷添加显著增加豆科草本植物

表1 氮、磷添加及其交互作用影响的土壤有效氮、磷含量和有效氮磷 比例(平均值土标准误)

Table 1 Effect of nitrogen $(\mathrm{N})$ and phosphorus $(\mathrm{P})$ addition and their interaction on available $\mathrm{N}$ and $\mathrm{P}$ concentration and available $\mathrm{N}: \mathrm{P}$ ratio in bulk soil $($ mean $\pm S E)$

\begin{tabular}{cccc}
\hline $\begin{array}{c}\text { 处理 } \\
\text { Treatments }\end{array}$ & $\begin{array}{c}\text { 有效氮 } \\
\text { Available N }\left(\mathrm{mg} \cdot \mathrm{kg}^{-1}\right)\end{array}$ & $\begin{array}{c}\text { 有效磷 } \\
\text { Available } \mathrm{P}\left(\mathrm{mg} \cdot \mathrm{kg}^{-1}\right)\end{array}$ & $\mathrm{N}: \mathrm{P}$ \\
\hline Control & $37.05 \pm 0.50$ & $5.08 \pm 0.07^{\mathrm{a}}$ & $7.31 \pm 0.13^{\mathrm{c}}$ \\
$\mathrm{N}$ & $72.02 \pm 1.15^{\mathrm{a}}$ & $5.11 \pm 0.05^{\mathrm{a}}$ & $14.12 \pm 0.25^{\mathrm{a}}$ \\
$\mathrm{P}$ & $37.65 \pm 0.64^{\mathrm{b}}$ & $9.36 \pm 0.11^{\mathrm{b}}$ & $4.03 \pm 0.07^{\mathrm{d}}$ \\
$\mathrm{NP}$ & $71.91 \pm 1.02^{\mathrm{a}}$ & $9.18 \pm 0.09^{\mathrm{b}}$ & $7.84 \pm 0.38^{\mathrm{b}}$ \\
\hline
\end{tabular}

Control, 无肥料添加; $N$, 氮添加; $P$, 磷添加; NP, 氮磷耦合添加。

Control, no fertiliser addition; N, nitrogen addition; $\mathrm{P}$, phosphorus addition; NP, coupling nitrogen and phosphorus addition. 

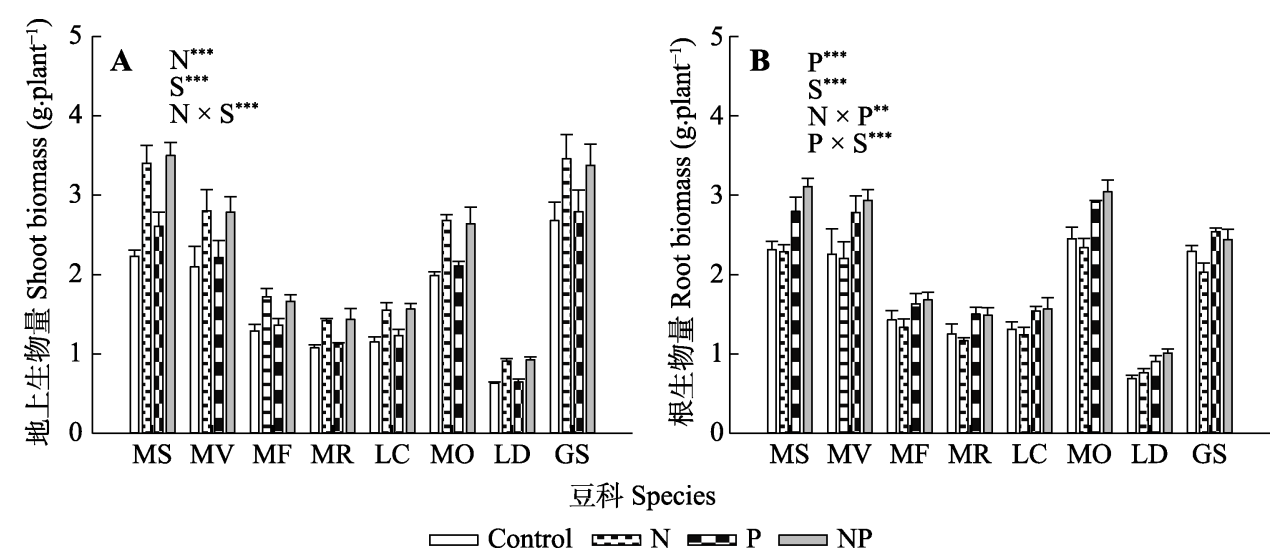

图1 氮、磷添加及其交互作用对不同豆科草本植物地上生物量(A)和根生物量(B)(平均值+标准误)的影响。Control, 无养分 添加; $\mathrm{N}$, 氮添加; $\mathrm{P}$, 磷添加; $\mathrm{NP}$, 氮磷耦合添加; $\mathrm{S}$, 物种。 GS, 野大豆; LC, 百脉根; $\mathrm{LD}$, 兴安胡枝子; MF, 黄花苜宿; MO, 草 木樨; MR，花苜蕧; MS，紫花苜㑑; MV，杂花苜宿。星号表示经一般线性模型统计分析得因子效应具有显著性。***, $p<0.001$; **, $p<0.01 ; *, p<0.05$ 。

Fig. 1 Influence of nitrogen $(\mathrm{N})$ and phosphorus $(\mathrm{P})$ addition and their interaction on shoot biomass $(\mathbf{A})$ and root biomass $(\mathbf{B})$ (mean $+S E$ ) of different herbaceous legumes. Control, no nutrient addition; N, N addition; $\mathrm{P}, \mathrm{P}$ addition; NP, coupled addition of $\mathrm{N}$ and $\mathrm{P} ; \mathrm{S}$, species. GS, Glycine soja; LC, Lotus corniculatus; LD, Lespedeza daurica; MF, Medicago falcata; MO, Melilotus officinalis; MR, Medicago ruthenica; MS, Medicago sativa; MV, Medicago varia. Asterisks indicate that factor effect was significant by general linear model analysis. ***, $p<0.001 ; * *, p<0.01 ; *, p<0.05$.
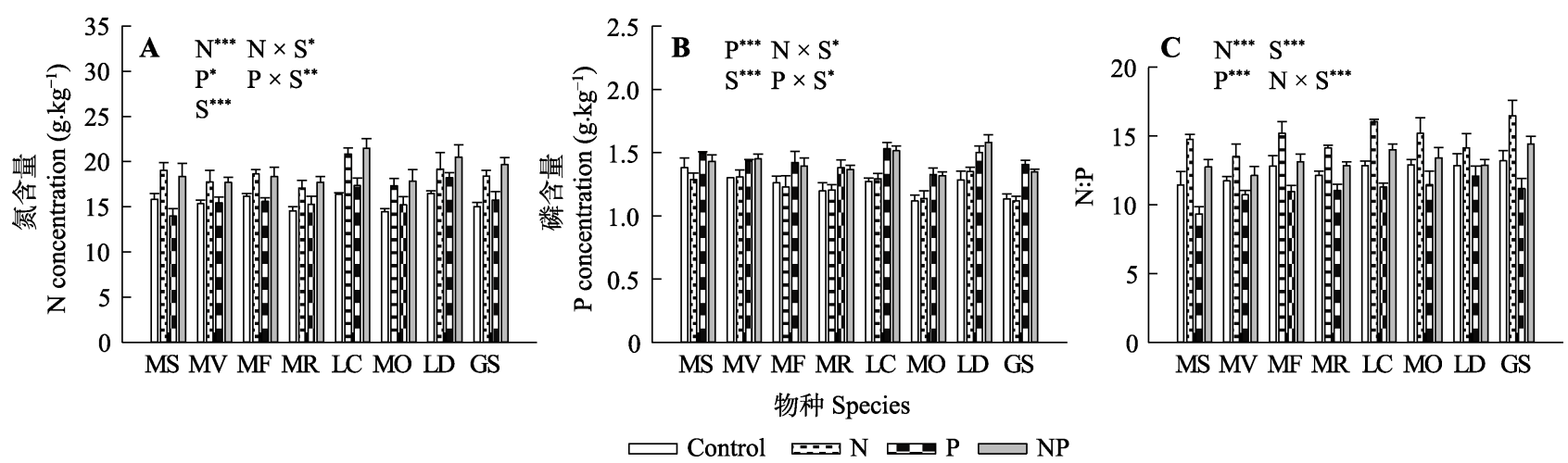

图2 氮、磷添加及其交互作用对不同豆科草本植物氮含量 $(\mathbf{A})$ 、磷含量 $(\mathbf{B})$ 和氮磷比 $(\mathbf{C})$ (平均值+标准误)的影响。Control, 无 养分添加; $\mathrm{N}$, 氮添加; P, 磷添加; $\mathrm{NP}$, 氮磷耦合添加; S, 物种。GS, 野大豆; LC, 百脉根; $\mathrm{LD}$, 兴安胡枝子; MF, 黄花苜宿; MO, 草木灖; MR, 花苜宿; MS, 紫花苜宿; MV, 杂花苜宿。星号表示经一般线性模型统计分析得因子效应具有显著性。***, $p<$ $0.001 ; * *, p<0.01 ; *, p<0.05$ 。

Fig. 2 Influence of nitrogen $(\mathrm{N})$ and phosphorus $(\mathrm{P})$ addition and their interaction on plant $\mathrm{N}$ concentration $(\mathbf{A})$, plant $\mathrm{P}$ concentration $(\mathbf{B})$ and plant N:P ratio $(\mathbf{C})($ mean $+S E)$ of different herbaceous legumes. Control, no nutrient addition; N, N addition; P, P addition; NP, coupled addition of N and P; S, species. GS, Glycine soja; LC, Lotus corniculatus; LD, Lespedeza daurica; MF, Medicago falcata; MO, Melilotus officinalis; MR, Medicago ruthenica; MS, Medicago sativa; MV, Medicago varia. Asterisks indicate that factor effect was significant by general linear model analysis. ${ }^{* * *}, p<0.001 ;{ }^{* *}, p<0.01 ; *, p<0.05$.

根际土壤 $\mathrm{pH}$ 。相反, 无磷/磷添加下, 氮添加平均促 使豆科草本植物根际柠檬酸含量增加 $20 \%$ 或 $4 \%$, 磷 添加显著降低豆科草本植物根际柠檬酸含量(图4D)。

对于所有豆科草本植物, 平均根生物量及根非 结构性碳水化合物含量与根部菌根侵染率、根际柠 檬酸含量存在负相关关系, 然而, 平均根生物量及 根非结构性碳水化合物含量与根际土壤 $\mathrm{pH}$ 存在显 著正相关关系(图5)。

\section{4 根瘤发育和生物固氮率}

受限于磷资源的有效性和豆科物种, 氮添加降
低豆科草本植物根瘤数量和生物量, 在无磷/磷添 加下，氮添加导致豆科草本植物生物固氮率分别下 降了 $27 \%-47 \%$ 和 $16 \%-22 \%$, 综合两个氮添加处理, 磷添加促使 8 种豆科草本植物生物固氮率提高 $17 \%-57 \%$ (图6A、6B、6D)。然而，氮添加对根瘤的 磷含量并没有显著影响, 磷添加提高了豆科草本植 物根瘤磷含量(图6C)。

回归分析表明, 随着不同的养分添加, 根生物 量及其非结构性碳水化合物含量与豆科草本植物生 物固氮率间存在显著正相关关系(图7), 而豆科草本 


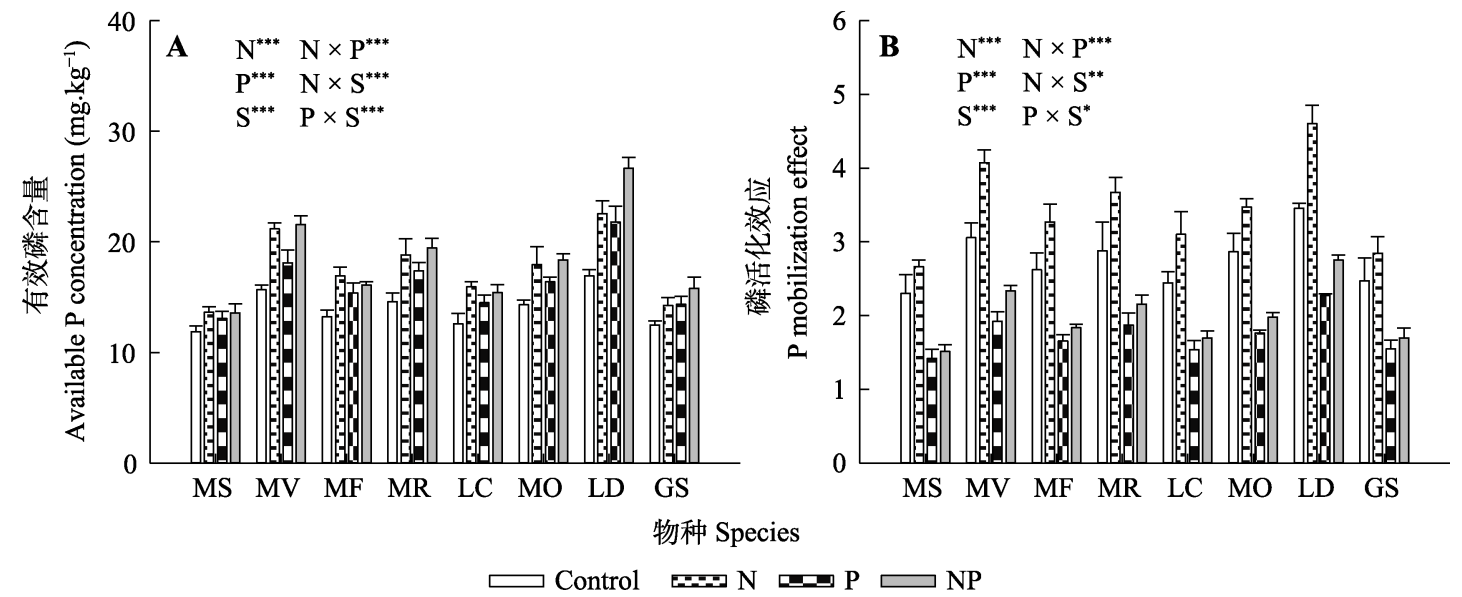

图3 氮、磷添加及其交互作用对不同豆科草本植物根际有效磷含量(A)和根际磷活化效应(B)(平均值+标准误)的影响。Control, 无养分添加; $N$, 氮添加; P, 磷添加; NP, 氮磷耦合添加; S, 物种。GS, 野大豆; LC, 百脉根; LD, 兴安胡枝子; MF, 黄花苜宿; MO, 草木樨; MR, 花苜葆; MS, 紫花苜宿; MV, 杂花苜宿。星号表示经一般线性模型统计分析得因子效应具有显著性。***, $p$ $<0.001 ; * *, p<0.01 ; *, p<0.05$ 。

Fig. 3 Influence of nitrogen $(\mathrm{N})$ and phosphorus $(\mathrm{P})$ addition and their interaction on available $\mathrm{P}$ concentration in rhizosphere (A) and $\mathrm{P}$ mobilization in rhizosphere $(\mathbf{B})($ mean $+S E)$ of different herbaceous legumes. Control, no nutrient addition; $\mathrm{N}, \mathrm{N}$ addition; $\mathrm{P}, \mathrm{P}$ addition; NP, coupled addition of N and P; S, species. GS, Glycine soja; LC, Lotus corniculatus; LD, Lespedeza daurica; MF, Medicago falcata; MO, Melilotus officinalis; MR, Medicago ruthenica; MS, Medicago sativa; MV, Medicago varia. Asterisks indicate that factor effect was significant by general linear model analysis. ${ }^{* * *}, p<0.001 ; * *, p<0.01 ; *, p<0.05$.
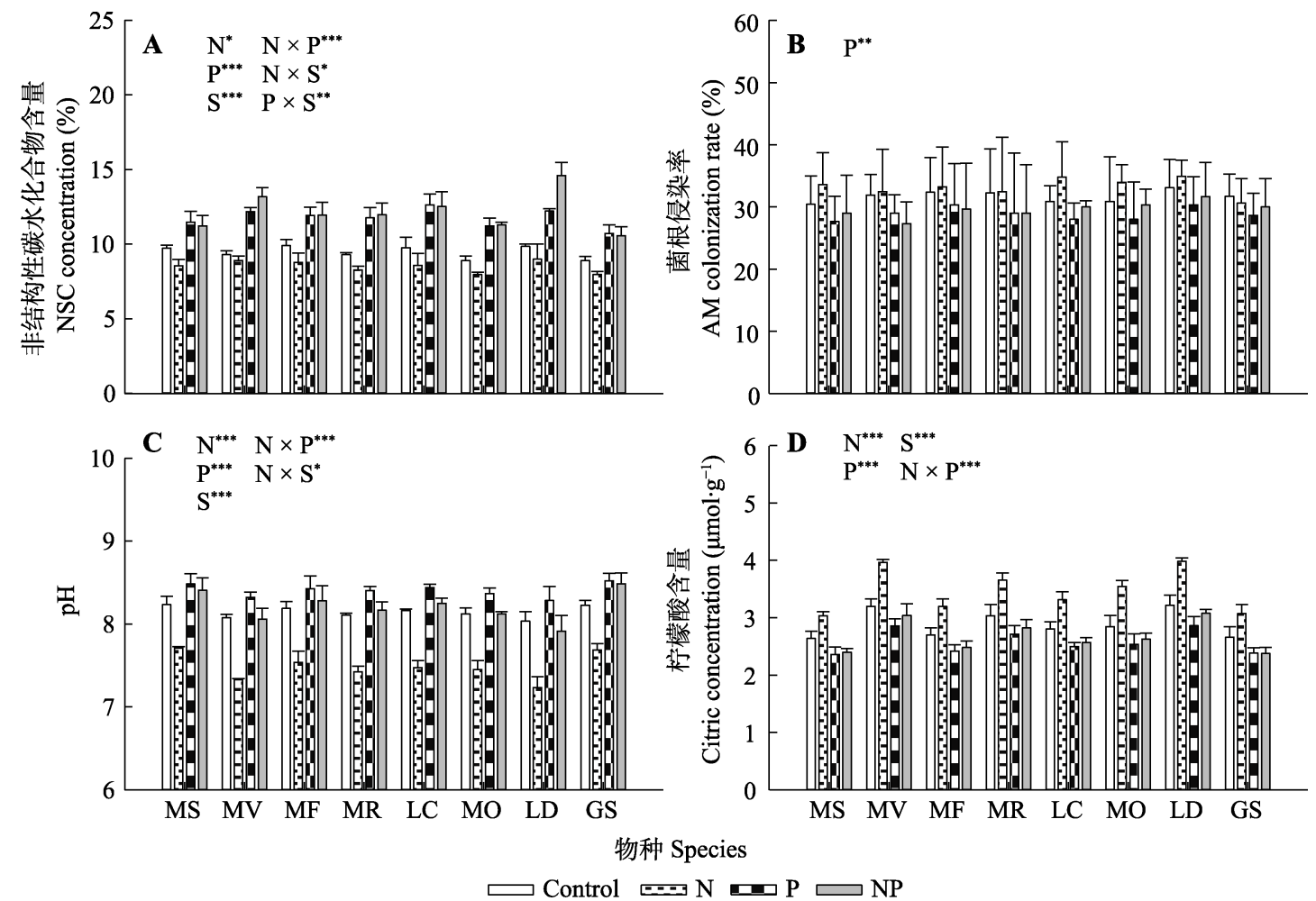

图4 氮、磷添加及其交互作用对不同豆科草本植物根系非结构性碳水化合物(NSC)含量 (A)、根系的菌根(AM)侵染率(B)、根 际 $\mathrm{pH}(\mathbf{C})$ 和根际柠檬酸含量 $(\mathbf{D})$ (平均值+标准误)的影响。Control, 无养分添加; $\mathrm{N}$, 氮添加; P, 磷添加; NP, 氮磷耦合添加; S, 物种。GS, 野大豆; LC, 百脉根; LD, 兴安胡枝子; MF, 黄花苜葆; MO, 草木樨; MR, 花苜宿; MS, 紫花苜宿; MV, 杂花苜芿。 星号表示经一般线性模型统计分析得因子效应具有显著性。***, $p<0.001 ; * *, p<0.01 ; *, p<0.05$ 。

Fig. 4 Influence of nitrogen (N) and phosphorus (P) addition and their interaction on non-structure carbohydrate (NSC) concentration in root (A), arbuscular mycorrhizal (AM) colonization rate of root (B), pH in rhizosphere (C) and citric concentration in rhizosphere $(\mathbf{D})($ mean $+S E)$ of different herbaceous legumes. Control, no nutrient addition; N, N addition; P, P addition; NP, coupled addition of N and P; S, species. GS, Glycine soja; LC, Lotus corniculatus; LD, Lespedeza daurica; MF, Medicago falcata; MO, Melilotus officinalis; MR, Medicago ruthenica; MS, Medicago sativa; MV, Medicago varia. Asterisks indicate that factor effect was significant by general linear model analysis. ${ }^{* * *}, p<0.001 ;{ }^{* *}, p<0.01 ;{ }^{*}, p<0.05$. 
植物根际的磷活化效应与其生物固氮率间存在显著 负相关关系(图8)。

\section{3 讨论}

\section{1 根系生物量和根部非结构性碳水化合物含量 的下降导致豆科草本植物固氮抑制}

生物固氮和吸收土壤氮是豆科植物两个可供选 择的氮获取策略(Ledgard et al., 2001)。如果排除其 他环境限制, 土壤氮资源的状态是豆科植物氮获取 策略选择的关键决定因素(West et al., 2005)。氮添加 提高了土壤氮资源有效性, 可能降低豆科植物为获 取大气氮而对根和根瘤发育的投资。再者, 增加的 土壤氮有效性可能将豆科草本植物间竞争的压力从 地下转移到地上(Farrer \& Suding, 2016), 诱使植物 投资更多资源到地上生物量用以捕获光, 并因此限 制根系发育(Aerts et al., 1991)。本研究中, 对比无肥 料添加, 氮添加提高了地上生物量, 并小幅降低根 生物量, 已经表明了氮添加下豆科草本植物生物量 分配的转移。同时, 我们的研究也发现氮添加降低了
豆科草本植物根部非结构性碳水化合物的含量, 间 接地表明土壤氮资源的增加可能改变了光合产物的 分配，促使豆科草本植物将更多的光合产物转移并储 存在地上，并导致根部碳水化合物营养储存的下降。

实际上，本研究发现氮有效性并不是影响豆科 草本植物根生物量和根非结构性碳水化合物的独立 因素，因为磷对氮添加效应有显著的调控作用(图 $1 \mathrm{~B}$ ，图4A)。由增加的根际磷活化能够判断氮添加加 剧了植物磷营养限制, 这一过程很可能通过改变豆 科草本植物根部磷代谢生理影响根生物量和非结构 性碳水化合物含量。而根据本研究结果, 磷活化的 增加也可能同样降低根生物量和根非结构性碳水化 合物含量(图5)。豆科植物根系为根瘤细菌提供了共 生位点, 并将碳水化合物等养分输送至根瘤支持其 生长代谢(Ledgard \& Steele, 1992), 因此豆科植物根 系的生物量和碳水化合物含量对根瘤固氮有重要的 调控作用, 本研究的结果已经发现根生物量、根碳 水化合物含量与豆科草本植物生物固氮率间存在显
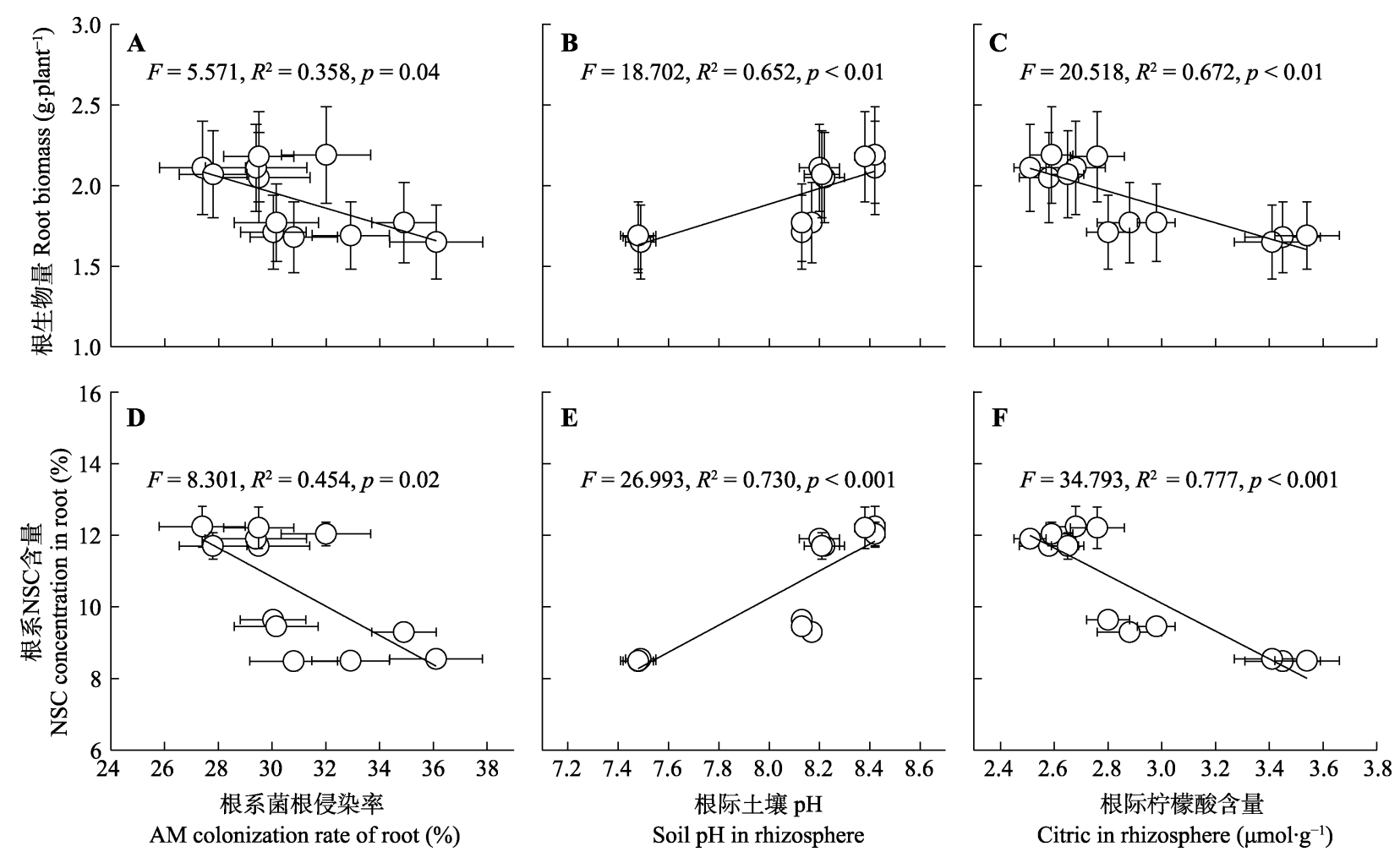

图5 不同养分添加下 8 种豆科草本植物平均根生物量与根系菌根 $(\mathrm{AM})$ 侵染率 $(\mathbf{A})$ 、根际土壤 $\mathrm{pH}(\mathbf{B})$ 、根际柠檬酸含量 $(\mathbf{C})$ 间相 关关系, 及平均根系非结构性碳水化合物 $(\mathrm{NSC})$ 含量与 $\mathrm{AM}$ 侵染率 $(\mathbf{D}) 、$ 根际土壤 $\mathrm{pH}(\mathbf{E}) 、$ 根际柠檬酸含量 $(\mathbf{F})$ (平均值土标准误) 间相关关系。

Fig. 5 Correlation relationships between mean root biomass and arbuscular mycorrhizal (AM) colonization rate of root $(\mathbf{A})$, soil $\mathrm{pH}$ in rhizosphere (B), citric concentration in rhizosphere (C), and between mean non-structure carbohydrate (NSC) concentration in root and $\mathrm{AM}$ colonization rate of root $(\mathbf{D})$, soil $\mathrm{pH}$ in rhizosphere $(\mathbf{E})$, citric concentration in rhizosphere $(\mathbf{F})(\mathrm{mean} \pm S E)$ following different nutrient addition treatments on eight herbaceous legumes. 

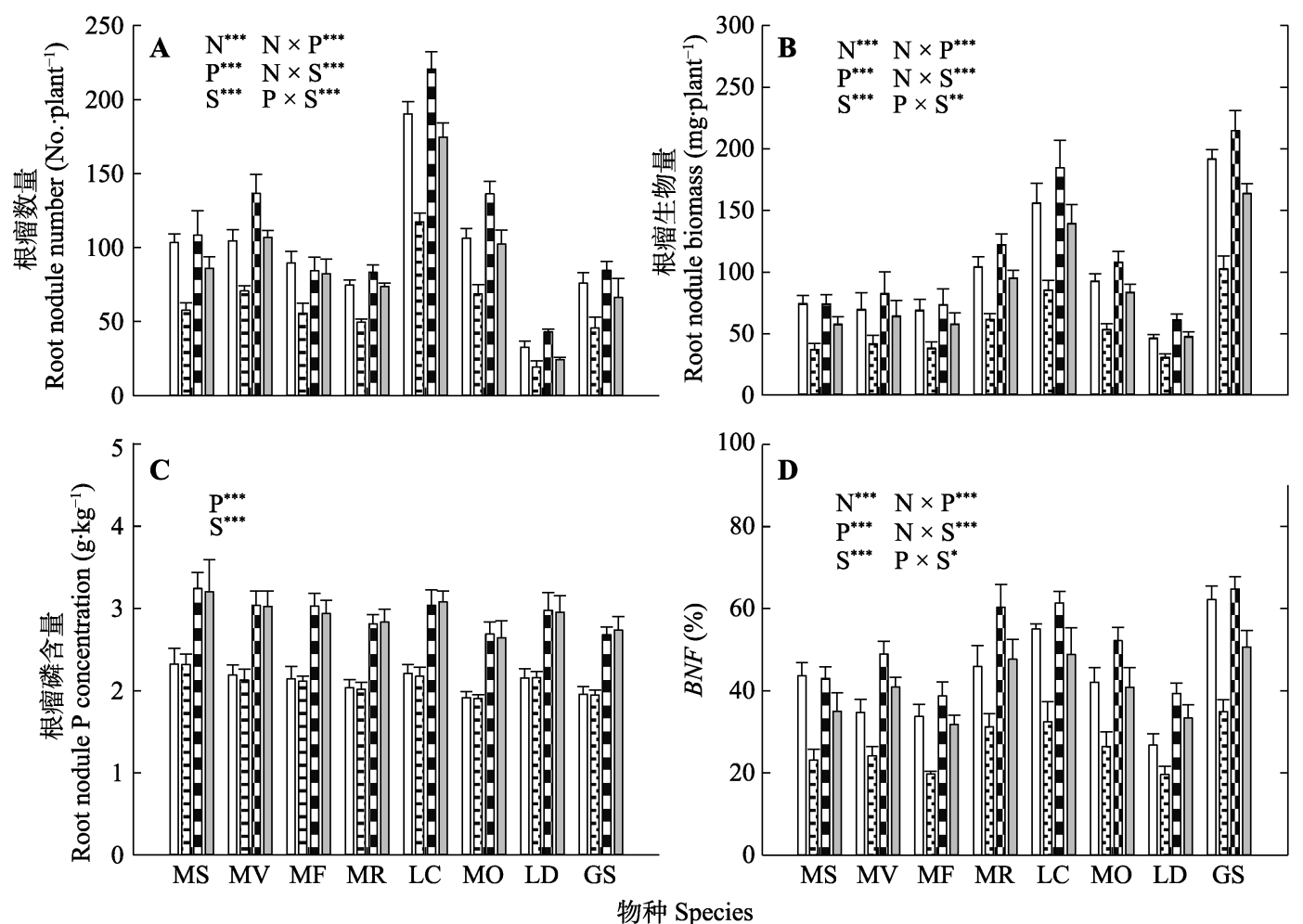

Control $\because \mathrm{N} \cong \mathrm{P} \rightleftharpoons \mathrm{NP}$

图6 氮、磷添加及其交互作用对不同豆科草本植物根瘤数目 $(\mathbf{A})$ 、根瘤生物量 $(\mathbf{B}) 、$ 根瘤磷含量 $(\mathbf{C})$ 和生物固氮率 $(B N F)(\mathbf{D})($ 平 均值+标准误)的影响。Control, 无养分添加; N, 氮添加; P, 磷添加; NP, 氮磷耦合添加; S, 物种。GS, 野大豆; LC, 百脉根; LD, 兴安胡枝子; MF, 黄花苜宿; MO, 草木樨; MR, 花苜宿; MS, 紫花苜宿; MV, 杂花苜宿。星号表示经一般线性模型统计分析得 因子效应具有显著性。***, $p<0.001 ; * *, p<0.01 ; *, p<0.05$ 。

Fig. 6 Influence of nitrogen $(\mathrm{N})$ and phosphorus $(\mathrm{P})$ addition and their interaction on root nodule number $(\mathbf{A})$, root nodule biomass $(\mathbf{B})$, root nodule $\mathrm{P}$ concentration $(\mathbf{C})$ and biological $\mathrm{N}$ fixation rate $(B N F)(\mathbf{D})($ mean $+S E)$ of different herbaceous legumes. Control, no nutrient addition; N, N addition; P, P addition; NP, coupled addition of N and P; S, species. GS, Glycine soja; LC, Lotus corniculatus; LD, Lespedeza daurica; MF, Medicago falcata; MO, Melilotus officinalis; MR, Medicago ruthenica; MS, Medicago sativa; $\mathrm{MV}$, Medicago varia. Asterisks indicate that factor effect was significant by general linear model analysis. ${ }^{* * *}, p<0.001 ; * *, p<$ $0.01 ; *, p<0.05$
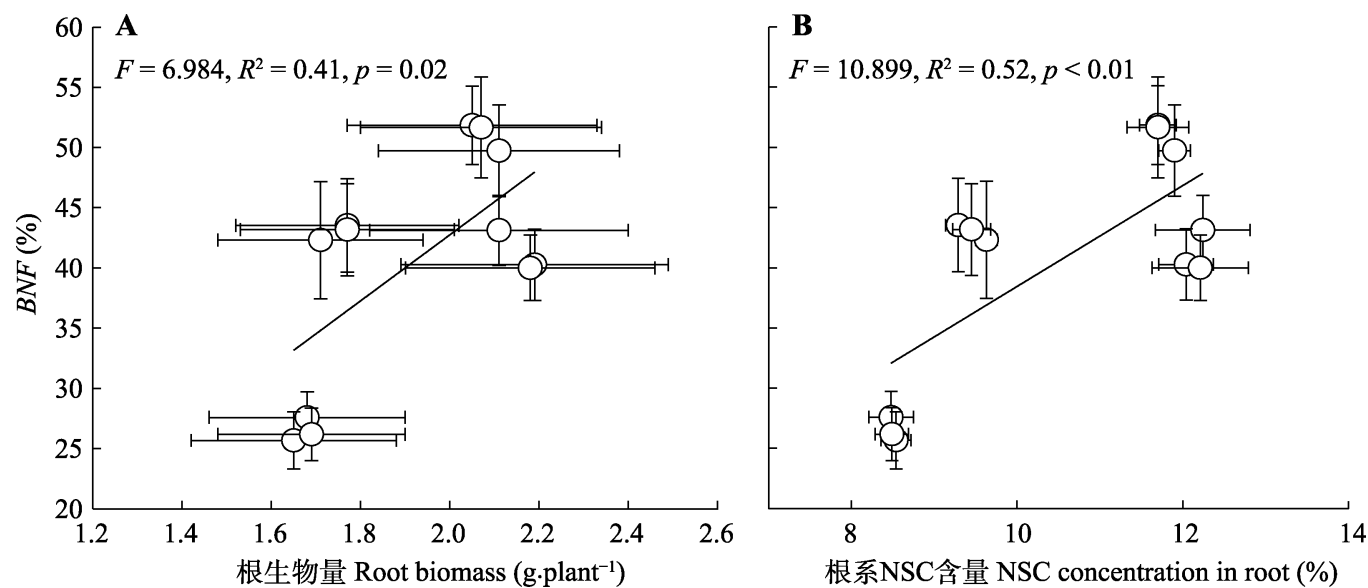

图7 不同养分添加下8种豆科草本植物平均根生物量(A)、根非结构性碳水化合物(NSC)含量(B)与豆科草本植物生物固氮率 $(B N F)$ (平均值土标准误)间相关关系。

Fig. 7 Correlation relationships between root biomass (A), root non-structure carbohydrate (NSC) concentration (B) and biological nitrogen fixation of legume $(B N F)($ mean $\pm S E)$ following different nutrient addition treatments on eight herbaceous legumes.

著的正相关关系(图7)。上述讨论表明不管单独的氮 增加, 还是相对的磷限制, 氮添加都能够通过降低
根生物量和根系碳水化合物含量降低豆科草本植物 的生物固氮率。

www.plant-ecology.com 


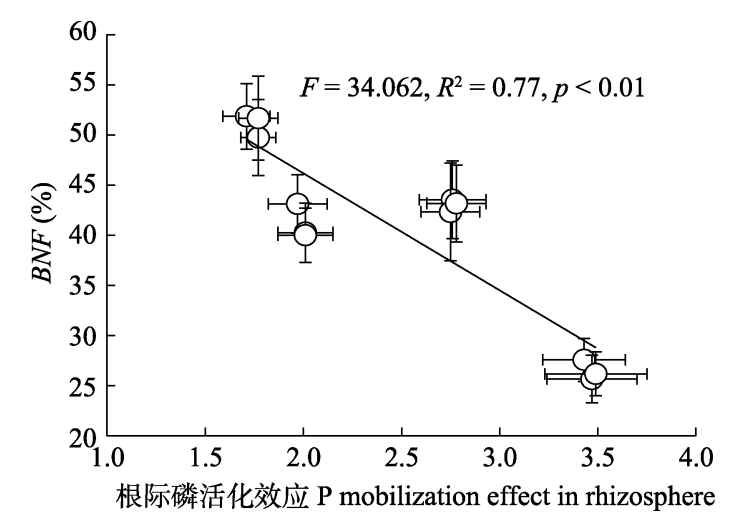

图8 不同养分添加下 8 种豆科草本植物平均根际磷活化效 应与豆科草本植物生物固氮率 $(B N F)$ (平均值沶准误)间相关 关系。

Fig. 8 Correlation relationships between phosphorus (P) mobilization effect in rhizosphere and biological nitrogen fixation rate of legume $(B N F)($ mean $\pm S E)$ following different nutrient addition treatments on eight herbaceous legumes.

\section{2 氮增加下豆科草本植物生物固氮和磷吸收的 权衡}

本实验中所用土壤磷含量较低, 可能已经限制 了豆科草本植物的生长(Betencourt et al., 2012), 这 一推断能够从控制盆中已经较高的根际磷活化效应 (图3B)得以佐证。氮添加显著提高了豆科草本植物 生物量和组织氮含量, 因为植物生长和代谢过程对 氮、磷元素的匹配需求, 氮添加将潜在提高植物对 磷的需要(White \& Hammond, 2008; Png et al., 2017)。然而, 当前的研究发现氮添加并未改变土壤 中磷的供给。而这种氮磷供应的不平衡并未导致豆 科草本植物降低植物和根瘤的磷含量, 反而激发了 豆科草本植物根际磷的活化和吸收, 以满足增加的 磷需求(图4)(Nuruzzaman et al., 2006; Betencourt et al., 2012)。因为植物的养分吸收主要发生在根际 (Hinsinger, 1998), 植物物种已经进化发展了一系列 策略来提高根际磷吸收。已知的策略包括加强根系菌根互作以提高根部磷吸收的范围和面积(Smith \& Read, 2008); 或通过根际土壤酸化刺激钙结合无机 磷的活化, 以增加根际有效磷数量, 这种作用在中 性或类似于本研究的碱性土壤中可能尤为重要 (Richardson et al., 2009; Betencourt et al., 2012)。此 外, 植物根部能够分泌一些磷活化有机物, 如柠檬 酸等有机酸, 这些磷活化物质也能提高根际磷有效 性(Nuruzzaman et al., 2006)。当前研究发现氮添加 下, 根际土壤 $\mathrm{pH}$ 显著下降, 而根部菌根侵染率明显 增加, 这些结果表明氮添加已经激发了豆科草本植
物多途径的磷截获策略。众所周知, 菌根的共生依 赖于根部提供碳水化合物营养(Soper et al., 2019)。 此外, 根际土壤 $\mathrm{pH}$ 的下降一般得益于根呼吸和根际 微生物呼吸的增加, 而增加的根呼吸和根际微生物 呼吸提高了根内及其分泌到根际的碳水化合物的消 耗(Hinsinger et al., 2003; Nuruzzaman et al., 2006)。 因此, 通过菌根共生和根际磷活化的途径来获取更 多磷可能潜在降低根生物量和根碳水化合物的积累 (图5)。本研究中根生物量、根碳水化合物和豆科草 本植物生物固氮率间存在正相关关系, 表明氮添加 下, 豆科草本植物为提高磷吸收, 会抑制其生物固 氮率。

最近的meta分析发现, 氮增加对生物固氮的作 用随生态系统类型、地理位置、气候条件等的变化 而发生改变(Zheng et al., 2019)。实际上这些大尺度 环境因子可能已经通过改变土壤养分背景而调控了 氮增加对生物固氮的影响。先前的研究发现氮增加 有时并不下调豆科植物生物固氮率, 并将其归因为 该生态系统具有较低的土壤氮水平, 导致该生态系 统即使在氮增加情境下也处于持续的氮限制状态 (Reed et al., 2007)。通过本研究, 我们进一步指出土 壤磷有效性调控 $\mathrm{N}: \mathrm{P}$ 平衡, 对氮增加下的豆科草本 植物生物固氮率具有重要调节作用(Gentili \& Huss-Danell, 2003)。实际上, 在陆地生态系统中, 磷 对植物生长的限制可能远比之前认定的要普遍, 尤 其在人类诱导的不平衡的氮、磷输入情景下 (Mahowald et al., 2008; Vitousek et al., 2010; Li et al., 2016b), 由此磷对豆科植物生物固氮的调控功能将 变得越来越重要。

\section{3 磷添加缓解氮富集对豆科草本植物生物固氮 的抑制作用}

磷添加能够促进豆科植物生长和生物固氮 (Augusto et al., 2013; Maistry et al., 2013), 主要有两 个原因。首先, 磷是固氮豆科植物及其根瘤细胞构 建的主要元素, 并且是生物固氮过程中关键的能量 驱动要素(Sulieman et al., 2013; Divito \& Sadras, 2014)。此外, 磷的添加也促进豆科植物生长, 并相 应增加其对氮的需求, 由此刺激根瘤生物量和生物 固氮的增加以满足氮需要(Batterman et al., 2013)。 本研究结果证实磷添加显著提高豆科草本植物生物 固氮率。进一步地, 本研究阐述了磷添加促进豆科 草本植物固氮的一个新机制, 即磷添加降低豆科草 
本植物根系-菌根互作和根际磷活化, 因此促进根生 物量和根碳水化合物的累积, 间接提高生物固氮率。

更大的氮有效性提高植物磷需求, 增加了土壤 磷对豆科草本植物生长和固氮的限制(Vitousek et al., 2010; Png et al., 2017)。因此, 氮富集下补充磷资 源对缓解生物固氮的磷限制尤为重要。这一推断被 我们的结果所支持, 因为本研究发现氮添加情境下 补充磷对豆科草本植物生物固氮率有更大的提升。 目前, 由于不成比例的氮磷输入 $(\mathrm{N}>\mathrm{P})$, 全球变 化和农业活动正在改变许多生态系统的氮磷平衡 (Vitousek et al., 2010)。根据我们的研究, 这样的氮 富集可能导致豆科植物生物固氮发生不同程度的改 变, 其变化程度取决于土壤磷状态。生态系统中磷有 效性越低, 氮添加对生物固氮的抑制效应越大。因此, 在高土壤 $\mathrm{N}: \mathrm{P}$ 或者高氮沉降速率的环境下, 为增加 豆科草本植物生物固氮, 补充磷是明智的选择。本 研究中, 平均所有豆科物种, 对比无营养添加, 氮和 磷协同添加并未改变豆科草本植物生物固氮率(对照: $41 \%$ vs. 氮磷添加: $43 \%$ ), 这一结果表明 $3.5 \mathrm{~g} \cdot \mathrm{m}^{-2}$ 的 磷添加能够有效缓解 $14 \mathrm{~g} \cdot \mathrm{m}^{-2}$ 的氮添加对实验豆科 草本植物生物固氮的抑制效应。这一结果对本研究 区豆科草场的优化管理具有重要指导意义。

\section{4 结论}

氮增加下, 松嫩草甸主要豆科草本植物生物固 氮率降低, 其潜在机制为氮增加提高了植物磷需求, 豆科草本植物为获取更多磷刺激根际酸化和根系菌根互作, 因此增加了根部碳水化合物的消耗, 导 致根生物量和碳水化合物累积的降低, 并由此限制 了根瘤生长及其功能。相反, 磷肥的添加缓解了豆 科草本植物的磷限制, 并通过促进根生物量和根碳 水化合物的累积提高生物固氮率, 其效应在氮添加 下尤为明显。

\section{参考文献}

Aerts R, Boot RGA, van der Aart PJM (1991). The relation between above- and belowground biomass allocation patterns and competitive ability. Oecologia, 87, 551-559.

Augusto L, Delerue F, Gallet-Budynek A, Achat DL (2013). Global assessment of limitation to symbiotic nitrogen fixation by phosphorus availability in terrestrial ecosystems using a meta-analysis approach. Global Biogeochemical Cycles, 27, 804-815.

Bai Y, Wu J, Clark CM, Naeem S, Pan Q, Huang J, Zhang L,
Han XG (2010). Tradeoffs and thresholds in the effects of nitrogen addition on biodiversity and ecosystem functioning: evidence from Inner Mongolia grasslands. Global Change Biology, 16, 358-372.

Batterman SA, Hedin LO, van Breugel M, Ransijn J, Craven DJ, Hall JS (2013). Key role of symbiotic dinitrogen fixation in tropical forest secondary succession. Nature, 502, 224-227.

Baziramakenga R, Simard RR, Leroux GD (1995). Determination of organic acids in soil extracts by ion chromatography. Soil Biology \& Biochemistry, 27, 349-356.

Betencourt E, Duputel M, Colomb B, Desclaux D, Hinsinger P (2012). Intercropping promotes the ability of durum wheat and chickpea to increase rhizosphere phosphorus availability in a low P soil. Soil Biology \& Biochemistry, 46, 181-190.

Buysse J, Merckx R (1993). An improved colorimetric method to quantify sugar content of plant tissue. Journal of Experimental Botany, 44, 1627-1629.

Divito GA, Sadras VO (2014). How do phosphorus, potassium and sulphur affect plant growth and biological nitrogen fixation in crop and pasture legumes? A meta-analysis. Field Crops Research, 156, 161-171.

Farrer EC, Suding KN (2016). Teasing apart plant community responses to $\mathrm{N}$ enrichment: the roles of resource limitation, competition and soil microbes. Ecology Letters, 19, 1287-1296.

Galloway JN (1998). The global nitrogen cycle: changes and consequences. Environmental Pollution, 102, 15-24.

Gates CT, Wilson JR (1974). The interaction of nitrogen and phosphorus on the growth, nutrient status and nodulation of Stylosanthes humilis H.B.K. (townsville stylo). Plant and Soil, 41, 325-333.

Gentili F, Huss-Danell K (2003). Local and systemic effects of phosphorus and nitrogen on nodulation and nodule function in Alnus incana. Journal of Experimental Botany, 54, 2757-2767.

Güsewell S (2004). N:P ratios in terrestrial plants: variation and functional significance. New Phytologist, 164, 243-266.

Hinsinger P (1998). How do plant roots acquire mineral nutrients? Chemical processes involved in the rhizosphere. Advances in Agronomy, 64, 255-265.

Hinsinger P, Plassard C, Tang C, Jaillard B (2003). Origins of root-mediated $\mathrm{pH}$ changes in the rhizosphere and their responses to environmental constraints: a review. Plant and Soil, 248, 43-59.

Ledgard SF, Sprosen MS, Penno JW, Rajendram GS (2001). Nitrogen fixation by white clover in pastures grazed by dairy cows: temporal variation and effects of nitrogen fertilization. Plant and Soil, 229, 177-187.

Ledgard SF, Steele KW (1992). Biological nitrogen fixation in mixed legume/grass pastures. Plant and Soil, 141, 
137-153.

Li Q, Song Y, Li G, Yu P, Wang P, Zhou D (2015). Grass-legume mixtures impact soil N, species recruitment, and productivity in temperate steppe grassland. Plant and Soil, 394, 271-285.

Li Q, Yu P, Li G, Zhou D (2016a). Grass-legume ratio can change soil carbon and nitrogen storage in a temperate steppe grassland. Soil and Tillage Research, 157, 23-31.

Li Q, Zhang H, Huang Y, Zhou D (2020). Forage nitrogen yield and soil nitrogen in artificial grasslands with varied Medicago seedling proportion. Archives of Agronomy and Soil Science, 66, 110-125.

Li Y, Niu S, Yu G (2016b). Aggravated phosphorus limitation on biomass production under increasing nitrogen loading: a meta-analysis. Global Change Biology, 22, 934-943.

Mahowald N, Jickells TD, Baker AR, Artaxo P, Benitez-Nelson CR, Bergametti G, Bond TC, Chen Y, Cohen DD, Herut B, Kubilay N, Losno R, Luo C, Maenhaut W, McGee KA, Okin GS, Siefert RL, Tsukuda S (2008). Global distribution of atmospheric phosphorus sources, concentrations and deposition rates, and anthropogenic impacts. Global Biogeochemical Cycles, 22, GB4026. DOI: 10.1029/ 2008GB003240.

Maistry PM, Cramer MD, Chimphango SBM (2013). N and P colimitation of $\mathrm{N}_{2}$-fixing and $\mathrm{N}$-supplied fynbos legumes from the Cape Floristic Region. Plant and Soil, 373, 217-228.

Miller RH, Keeney DR (1982). Methods of Soil Analysis, Part 2. Chemical and Microbiological Properties, 2nd ed. American Society of Agronomy, Madison, USA.

Nuruzzaman M, Lambers H, Bolland MDA, Veneklaas EJ (2006). Distribution of carboxylates and acid phosphatase and depletion of different phosphorus fractions in the rhizosphere of a cereal and three grain legumes. Plant and Soil, 281, 109-120.

Nyfeler D, Huguenin-Elie O, Suter M, Frossard E, Lüscher A (2011). Grass-legume mixtures can yield more nitrogen than legume pure stands due to mutual stimulation of nitrogen uptake from symbiotic and non-symbiotic sources. Agriculture, Ecosystem \& Environment, 140, 155-163.

Olsen SR, Cole CV, Watanabe SS, Dean LA (1954). Estimation of Available Phosphorus in Soil by Extraction with Sodium Bicarbonate. United States Department of Agriculture, Washington DC.

Peñuelas J, Sardans J, Rivas-Ubach A, Janssens IA (2012). The human-induced imbalance between C, N and P in Earth's life system. Global Change Biology, 18, 3-6.

Png GK, Turner BL, Albornoz FE, Hayes PE, Lambers H, Laliberté E (2017). Greater root phosphatase activity in nitrogen-fixing rhizobial but not actinorhizal plants with declining phosphorus availability. Journal of Ecology, 105, 1246-1255.
Reed SC, Cleveland CC, Townsend AR (2007). Controls over leaf litter and soil nitrogen fixation in two lowland tropical rain forests. Biotropica, 39, 585-592.

Richardson AE, Barea JM, McNeill AM, Prigent-Combaret C (2009). Acquisition of phosphorus and nitrogen in the rhizosphere and plant growth promotion by microorganisms. Plant and Soil, 321, 305-339.

Salvagiotti F, Cassman KG, Specht JE, Walters DT, Weiss A, Dobermann A (2008). Nitrogen uptake, fixation and response to fertilizer $\mathrm{N}$ in soybeans: a review. Field Crops Research, 108, 1-13.

Schade JD, Kyle M, Hobbie SE, Fagan WF, Elser JJ (2003). Stoichiometric tracking of soil nutrients by a desert insect herbivore. Ecology Letters, 6, 96-101.

Smith SE, Read D (2008). Mycorrhizal Symbiosis. 3rd ed. Academic Press, London, UK.

Song YT, Zhou DW, Li Q, Wang P, Huang YX (2012). Leaf nitrogen and phosphorus stoichiometry in 80 herbaceous plant species of Songnen grassland in Northeast China. Chinese Journal of Plant Ecology, 36, 222-230. [宋彦涛, 周道玮, 李强, 王平, 黄迎新 (2012). 松嫩草地80种草 本植物叶片氮磷化学计量特征. 植物生态学报, 36 , 222-230.]

Soper FM, Nasto MK, Osborne BB, Cleveland CC (2019). Nitrogen fixation and foliar nitrogen do not predict phosphorus acquisition strategies in tropical trees. Journal of Ecology, 107, 118-126.

Sparks DL, Page AL, Helmke PA, Loeppert RH, Soltanpour PN, Tabatabai MA, Johnston CT, Sumner ME (1996). Methods of Soil Analysis. Part 3. Chemical Methods. Soil Science Society of America, Madison, USA.

Sulieman S, van Ha C, Schulze J, Phan Tran LS (2013). Growth and nodulation of symbiotic Medicago truncatula at different levels of phosphorus availability. Journal of Experimental Botany, 64, 2701-2712.

Unkovich M, Herridge D, Peoples M, Cadisch G, Boddey B, Giller K, Alves B, Chalk P (2008). Measuring Plant-associated Nitrogen Fixation in Agricultural Systems. Australian Centre for International Agricultural Research, Canberra, Australia.

Vitousek PM, Cassman K, Cleveland C, Crews T, Field CB, Grimm NB, Howarth RW, Marino R, Martinelli L, Rastetter EB, Sprent JI (2002). Towards an ecological understanding of biological nitrogen fixation//Boyer EW, Howarth RW. The Nitrogen Cycle at Regional to Global Scales. Kluwer Academic Publishers, Dordrecht, the Netherlands. 1-45.

Vitousek PM, Menge DNL, Reed SC, Cleveland CC (2013). Biological nitrogen fixation: rates, patterns and ecological controls in terrestrial ecosystems. Philosophical Transactions of the Royal Society B: Biological Sciences, 368, 20130119. DOI: 10.1098/rstb.2013.0119. 
Vitousek PM, Porder S, Houlton BZ, Chadwick OA (2010). Terrestrial phosphorus limitation: mechanisms, implications, and nitrogen-phosphorus interactions. Ecological Applications, 20, 5-15.

West JB, HilleRisLambers J, Lee TD, Hobbie SE, Reich PB (2005). Legume species identity and soil nitrogen supply determine symbiotic nitrogen-fixation responses to elevated atmospheric $\left[\mathrm{CO}_{2}\right]$. New Phytologist, 167, 523-530.

White PJ, Hammond JP (2008). Phosphorus nutrition of terrestrial plants//White PJ, Hammond JP. The Ecophysiology of Plant-Phosphorus Interactions. Springer, Dordrecht, the Netherlands. 51-81.

Wu N, Li Z, Liu H, Tang M (2015). Influence of arbuscular mycorrhiza on photosynthesis and water status of Populus cathayana Rehder males and females under salt stress. Acta Physiol Plant, 37, 183.

Zhan S, Wang Y, Zhu Z, Li W, Bai Y (2017). Nitrogen enrichment alters plant N:P stoichiometry and intensifies phos- phorus limitation in a steppe ecosystem. Environmental and Experimental Botany, 134, 21-32.

Zheng M, Zhou Z, Luo Y, Zhao P, Mo J (2019). Global pattern and controls of biological nitrogen fixation under nutrient enrichment: a meta-analysis. Global Change Biology, 25, 3018-3030.

Zhou LL, Cao J, Zhang FS, Li L (2009). Rhizosphere acidification of faba bean, soybean and maize. Science of the Total Environment, 407, 4356-4362.

Zou CM, Wang YQ, Liu Y, Zhang XH, Tang S (2015). Responses of photosynthesis and growth to weak light regime in four legume species. Chinese Journal of Plant Ecology, 39, 909-916. [邹长明, 王允青, 刘英, 张晓红, 唐杉 (2015). 四种豆科作物的光合生理和生长发育对弱光的 响应. 植物生态学报, 39, 909-916.]

责任编委: 吕晓涛 编辑: 赵 航 